\title{
Performance evaluation of a single mode optical fiber tip sensor for glucose detection
}

\author{
Nazirah Mohd Razali, Puteri Nadiah Syamimi Said Ja'afar, Sumiaty Ambran \\ Department of Electronic Systems Engineering, Malaysia-Japan International Institue of Technology, \\ Universiti Teknologi Malaysia, Malaysia
}

\begin{tabular}{l} 
Article Info \\
\hline Article history: \\
Received Jan 20, 2020 \\
Revised Mar 20, 2020 \\
Accepted Apr 12, 2020 \\
\hline
\end{tabular}

Keywords:

Fresnel's reflection

Linearity

Optical fiber tip sensor glucose

Sensitivity

\begin{abstract}
Glucose must be carefully regulated to ensure its safety and quality. Nowadays, chemists often face difficulty to measure glucose concentrations as the current methods for the analysis are expensive and require complex procedures which have reduced the interest among chemists to use these techniques. This study evaluated the performance of a single mode optical fiber tip sensor for glucose detection. The sensing mechanism mainly depends on the Fresnel's reflection that occurs at the flat fiber tip region while the sensor performance was evaluated based on its sensitivity and linearity. The sensor was tested in glucose solution at different concentration range; high range $(0 \%-14 \%)$, medium range $(0 \%-1.4 \%)$ and low range $(0 \%-0.14 \%)$. The sensor showed a good performance when sensing the glucose solution at high and medium concentration range with strong linearity of more than $90 \%$ and an average sensitivity of $0.0979 \mathrm{dBm} / \%$ and $0.1019 \mathrm{dBm} / \%$, respectively. However, the low concentration range showed no significant changes during sensing. Based on the findings obtained in this study, the optical fiber tip sensor has the potential to be utilized for glucose detection and suitable for high and medium concentration range measurement.
\end{abstract}

Copyright $@ 2020$ Institute of Advanced Engineering and Science. All rights reserved.

\section{Corresponding Author:}

Nazirah Mohd Razali

Department of Electronic Systems Engineering,

Malaysia-Japan International Institue of Technology,

Universiti Teknologi Malaysia,

54100 Kuala Lumpur, Malaysia

Email: nazirahmohdrazali@gmail.com

\section{INTRODUCTION}

Glucose $\left(\mathrm{C}_{6} \mathrm{H}_{12} \mathrm{O}_{6}\right)$ is a simple sugar which is made up of six carbon atoms (C) that are linked to a hydroxyl group $(\mathrm{OH})$. This type of sugar is soluble in water and provide a sweet taste [1]. In the food industry, glucose is mainly used as a sweetener to improve the taste of food [2]. Nutritionally, glucose provides energy to the human body especially to the brain and nervous system [3]. Glucose is critical to life, so its level in the body must be carefully regulated. There are two consequences for an unregulated glucose level. Elevated glucose level can cause hyperglycemia, but lack of glucose level can cause hypoglycaemia $[4,5]$. Extremes hyperglycemia and hypoglycemia can lead to diabetic emergencies [6]. The signs and symptoms of both conditions are quite similar such as staggering and an intoxicated appearance.

Currently, chemists face difficulties in analyzing glucose samples. Various techniques have been employed to analyze the concentration of this liquid food products such as high-performance liquid chromatography (HPLC) [7, 8], capillary electrophoresis (CE) [9] and Fourier transform infrared (FTIR) analysis [10]. These techniques are useful, versatile, and extremely precise when it comes to quantifying chemical components, but they have several drawbacks because they are time-consuming, expensive, require 
high maintenance and highly skilled operators to perform the corresponding chemical processes [11, 12]. The drawbacks have reduced the interest in employing these techniques in analyzing the samples.

Hence, this paper proposes an optical fiber tip sensor solve this matter. Optical fiber tip sensor has attracted attention in sensing field as it only requires a single step process to fabricate the sensor; by cleaving the fiber to produce a flat end sensing surface $[13,14]$. Recently, optical fiber tip sensor has been utilized for measuring sucrose and ethanol level [15], glycerol [14], benzene, methanol, acetone concentration [16] and temperature level using sodium chloride solution [17]. Based on these references, we determined that it is feasible to utilize the optical fiber tip sensor for glucose detection. Therefore, this works aims to evaluate the performance of a single mode optical fiber tip sensor for this purpose.

\section{RESEARCH METHODOLOGY}

\subsection{Sensing system development and sensor preparation}

The characterization setup for the proposed sensor using the optical fiber tip is shown in Figure 1. In the experiment, an amplified spontaneous emission (Fiberlabs - ASE FL7004) is used as a light source which can produce a broadband spectrum operating at infrared wavelength. In this context, the broadband spectrum source is required to observe if there are any significant wavelength changes apart from the optical power changes. The optical circulator is used to direct the Fresnel light (or transduced light) from the sensing region to optical spectrum analyzer (Yokogawa - AQ6370D) to monitor the sensing response. The sensor is placed and fixed on a glass slide to reduce any physical disturbance. Throughout the experiment, the analyte is dropped onto the sensing probe using a $100 \mu \mathrm{L}$ pipette. The sensor performance is established from the light spectrum response. At the end of each sample measurement, the sensor is repeatedly cleaned with DI water followed by a proper drying process to ensure that no residues from the previous sample remains. During the experiment, the concentration is raised from low to high concentrations, and the experiment is repeated for three cycles. All the mixtures are stirred for 10 minutes to ensure homogeneity

The optical fiber tip sensor was selected for measurement because only a single step fabrication is required; by cleaving the fiber. An SMF (Corning SMF-28e) $20 \mathrm{~cm}$ in length with the core and cladding diameters of $8.2 \mu \mathrm{m}$ and $125 \pm 0.7 \mu \mathrm{m}$, respectively were used to prepare the sensor. The SMF was chosen in this work because it had low light loss [18], low cost, compatible with the existing setup and can operate at NIR wavelength which was an eye-safe wavelength [19]. The small part of the fiber tip is stripped and cleaned using dry tissue and propanol, followed by DI water to remove any debris. The tip is cleaved to produce a flat end surface sensing region as shown in Figure 2. The well-cleaved fiber structure is imaged using a digital microscope (Dino-Lite AM4515T8). The black and white strip exists due to image magnification and light effect adjustment

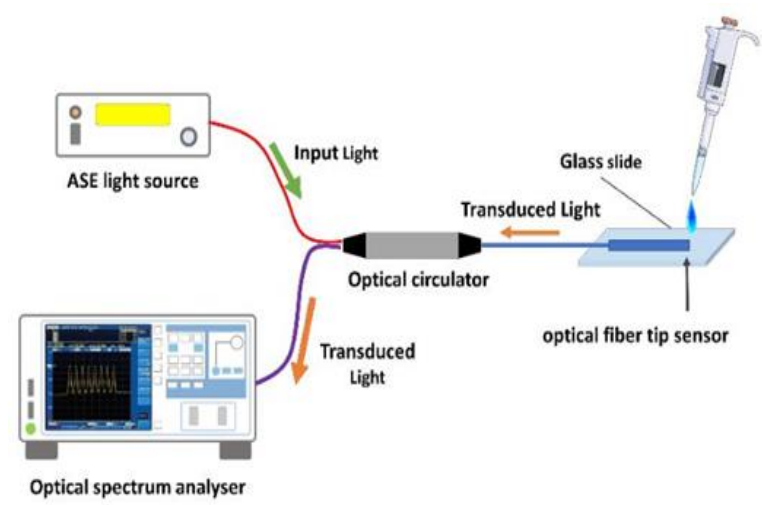

Figure 1. Characterization setup for glucose detection

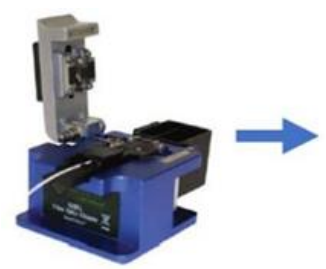

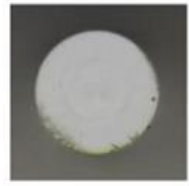

(a)

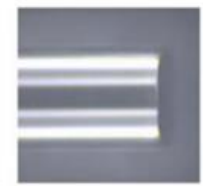

(b)
Figure 2. The optical fiber cleaving to produce the flat end fiber surface; (a) front view and (b) top view of the optical fiber tip sensor

\subsection{Sensing mechanism}

When the ASE light is injected into the analyte, Fresnel reflection occurs at the coupling interface between the fiber core and the analyte which has different refractive indices. The Fresnel light will be reflected into the core. The fraction of the optical power $(\mathrm{R})$ that is reflected on the interface is as in (1). 


$$
R=\left(\frac{n_{c}-n_{a}}{n_{c}+n_{a}}\right)^{2}
$$

Here, $\mathrm{n}_{\mathrm{c}}$ is the refractive index of the optical fiber core and $\mathrm{n}_{\mathrm{a}}$ is the refractive index of the contacted analyte with the core. Figure 3 illustrates the working principle of the optical fiber tip sensor used in this work. By changing $n_{a}$ with the different chemical composition of the analyte, Fresnel reflection arising at the interface between the tip of the optical fiber and the analyte is also changed.

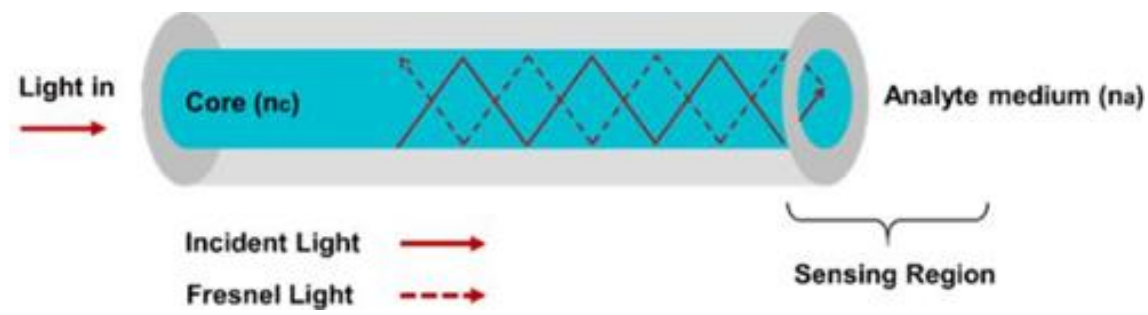

Figure 3. Working principle of the proposed optical fiber tip sensor

\subsection{Glucose sample preparation}

The procedure in preparing the glucose samples is illustrated in Figure 4. Initially, a concentrated solution is prepared before diluting the solution to obtain glucose at various concentration range. For instance, the concentrated glucose sample with $50 \%$ of concentration was prepared by adding $50 \mathrm{~g}$ of glucose powder in $50 \mathrm{~mL}$ of DI water. Next, the stock was diluted to obtain glucose at various concentrations ranging from high range: $0 \%-14 \%$, medium range: $0 \%-1.4 \%$ and low range: $0 \%-0.14 \%$. The concentration range used in this work was lower than those stated in previous work [20, 21]. The solution was diluted at a different range to determine the sensor's capabilities to measure smaller glucose concentration.

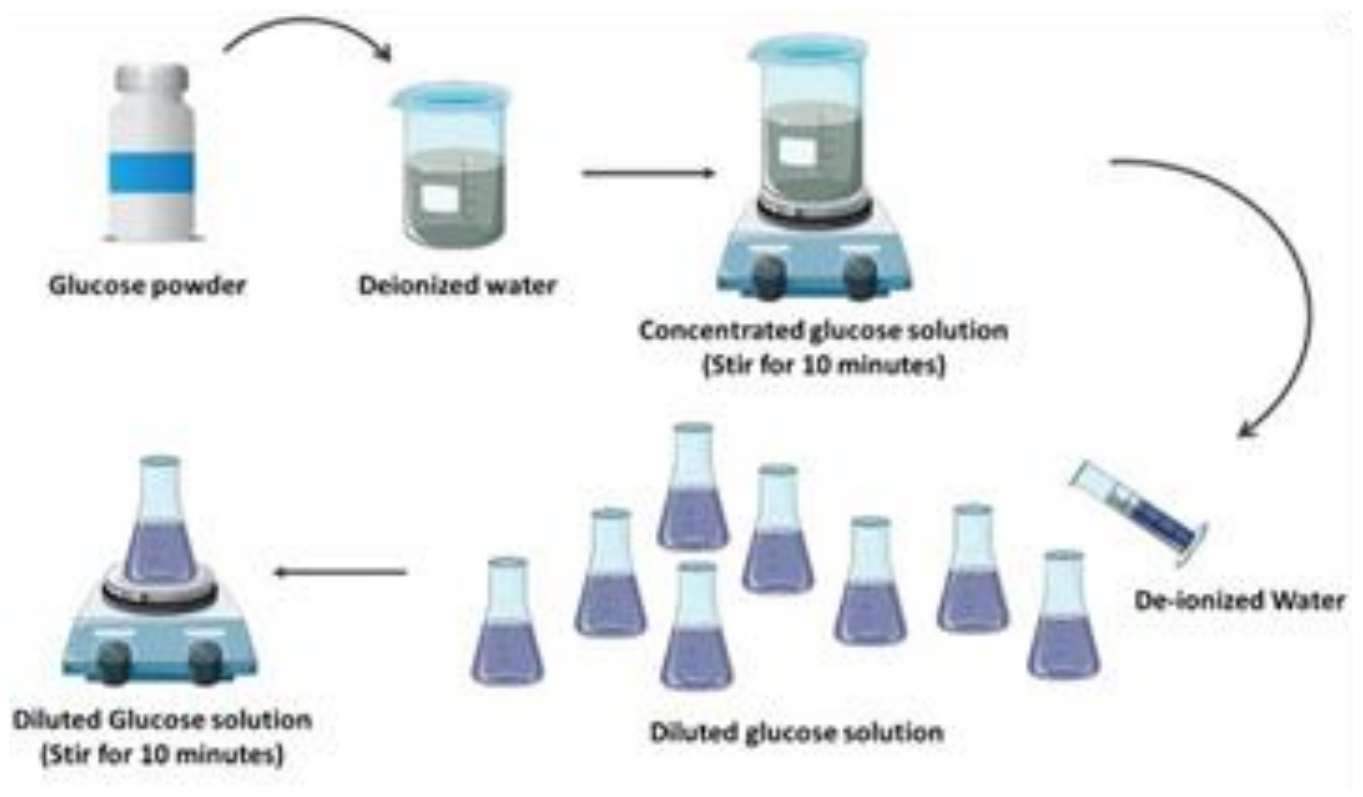

Figure 4. Glucose sample preparation

\subsection{Evaluation procedure}

The sensor performance was evaluated based on their two static characteristics: sensitivity and linearity. Sensitivity is the ratio of the change in the sensor's output $(\Delta y)$ to the change of the measurand in the input $(\Delta x)$. The slope of the graph curve, $y=f(x)$, can be used for the calculation of sensitivity [22]. The greater the graph slope value, the higher the sensitivity of the sensor. 
All the experiments were repeated for three cycles to evaluate the variations in the sensor's sensitivity. This can be performed by evaluating their standard deviations. An ideal standard deviation is zero $(0)$, meaning that there are no variations between the results. The more the standard deviations depart from zero, the more variations can be seen in the data distributions [23]. A low standard deviation means that most of the numbers are very close to the average [24], which indicates a constant sensitivity for the three repeated experiments. In contrast, a high standard deviation means that the numbers are spread out [24], which indicates that the sensitivity value fluctuated in the three repeated experiments. The formula for calculating the standard deviation is described in (2) [25]; $x$

$$
S=\sqrt{\frac{\sum(x-\bar{x})^{2}}{n-1}}
$$

Here, $\mathrm{s}$ is the standard deviation, $\mathrm{x}$ is the sensor sensitivity, $\overline{\mathrm{x}}$ is the average sensor sensitivity for the three repeated experiment and $\mathrm{n}$ is the number of repeated cycles. The closeness of the calibration curve to a specified straight line shows the linearity of a sensor. The linearity can be determined based on the regression coefficient (R2) from the plotted graph and given as (3) [26];

$$
\text { Linearity }=R^{2} \times 100 \%
$$

In this work, the sensor characteristics are set to a specific value to ensure its quality performance. The sensing response will be proclaimed as the good one if the sensor can provide a low standard deviation sensitivity value $(\mathrm{s} \leq 0.020)$ for the three repeated experiments with average linearity more than $90 \%$.

\section{RESULTS AND DISCUSSION}

The following analysis discusses the sensing response of the fiber tip sensor for all the three cycles of the experiment in the same condition. Since there are no significant wavelength changes during the measurement, all the data are analyzed at a fixed $1559 \mathrm{~nm}$ wavelength, based on the available peak value of ASE light source.

\subsection{Sensing response for glucose concentration at high range of $0 \%$ to $14 \%$}

Figure 5 depicts the glucose sensing spectrum and the analysis of the reflected light power of the glucose concentration range from $0 \%-14 \%$ for the first cycle of the experiment. In Figure 5(a), there are significant spectral changes shown by the proposed fiber tip sensor. In Figure 5(b), the optical power linearly decreases as the concentration of glucose increases. The initial reflected light of $-44.9533 \mathrm{dBm}$ is reduced to $46.3443 \mathrm{dBm}$, with a total power loss of $1.391 \mathrm{dBm}$. The sensitivity achieved by this sensor is 0.0979 $\mathrm{dBm} / \%$ with a linear regression value up to 0.9924 .

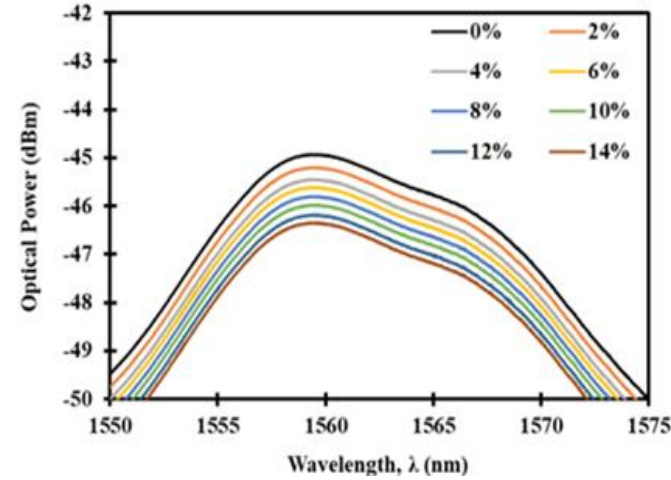

(a)

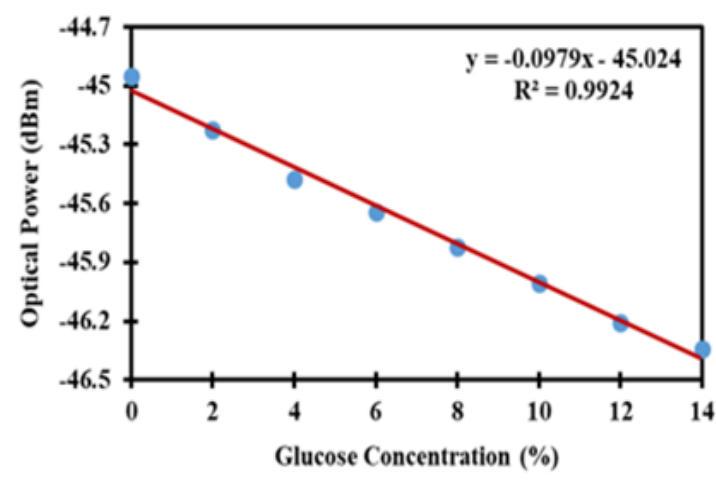

(b)

Figure 5. The sensing response; (a) sensing spectra and (b) analysis of reflected light intensity for the first cycle of the experiment at $1559 \mathrm{~nm}$ of wavelength for glucose solution range from $0 \%-14 \%$

Table 1 summarizes the sensor performance for a high range of glucose concentration for the three repeated cycles of the experiment. In the average analysis, the sensor sensitivity is $0.0979 \mathrm{dBm} / \%$ and the linearity is $99.27 \%$. The sensor sensitivity collected from the repeated cycles is evaluated based on the 
standard deviation. The evaluation is necessary to observe the variations in the results. The sensor sensitivity has a standard deviation of 0.000 which shows that there is no variation in sensitivity between the three repeated results. All the sensor sensitivity is very close to the average value which indicates that the sensor has a high possibility of producing a constant sensitivity for any repeated experiment.

Table 1. Optical fiber tip sensor performance at a high range of glucose concentration

\begin{tabular}{|c|c|c|c|}
\hline No. of Cycles & & & \\
\hline Sensor Sensitivity $(\mathrm{dBm} / \%)$ & 0.0979 & 0.0974 & 0.0970 \\
\hline Regression Coefficient, R2 & 0.9924 & 0.9892 & 0.9966 \\
\hline Average Sensitivity $(\mathrm{dBm} / \%)$ & & 0.0979 & \\
\hline Average Linearity (\%) & & 99.27 & \\
\hline Standard Deviation of the Sensitivity for Three Repeated Experiment, $s$ & & 0.000 & \\
\hline
\end{tabular}

\subsection{Sensing response for glucose concentration at medium range of $0 \%$ to $1.4 \%$}

Figure 6 depicts the glucose sensing spectrum and the analysis of the reflected light at a medium concentration range from $0 \%-1.4 \%$ for the first cycle of the experiment. As can be seen in Figure 6(a), there are also significant spectral changes shown by the proposed sensor. In Figure 6(b), the optical power is linearly decreasing with the increase in the glucose concentration. The initial light in the form of $-45.16 \mathrm{dBm}$ was reduced to $-45.28 \mathrm{dBm}$, giving a total loss of $0.12 \mathrm{dBm}$. The sensitivity achieved by this sensor for the first cycle experiment is $0.0821 \mathrm{dBm} / \%$ with a linear regression value of 0.9292 .

Table 2 shows the sensor performance during a medium range glucose concentration measurement for all the three cycles of the experiment. In the average analysis, the sensor sensitivity is $0.0950 \mathrm{dBm} / \%$ and the linearity value is $94.21 \%$. The standard deviation of the sensor sensitivity is 0.015 for the three repeated experiments, which is slightly higher than the high range concentration which indicates the sensor sensitivity results started to fluctuate in the three repeated experiments in the same condition. However, the standard deviation value still can be considered as a good value because the maximum acceptable value in this work is 0.020 .

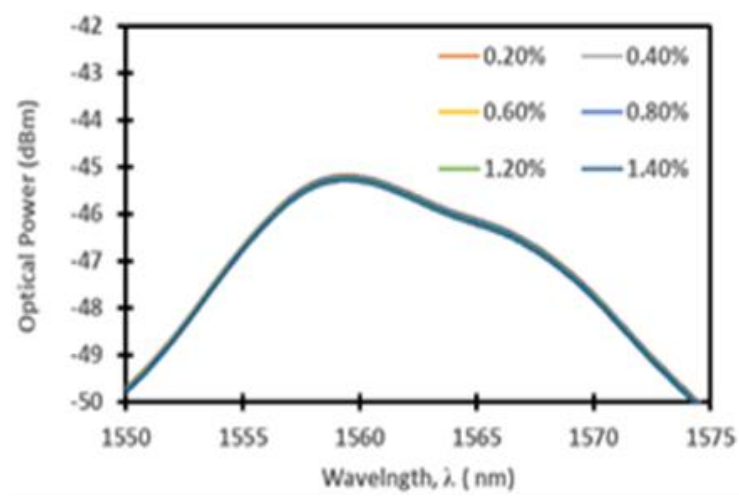

(a)

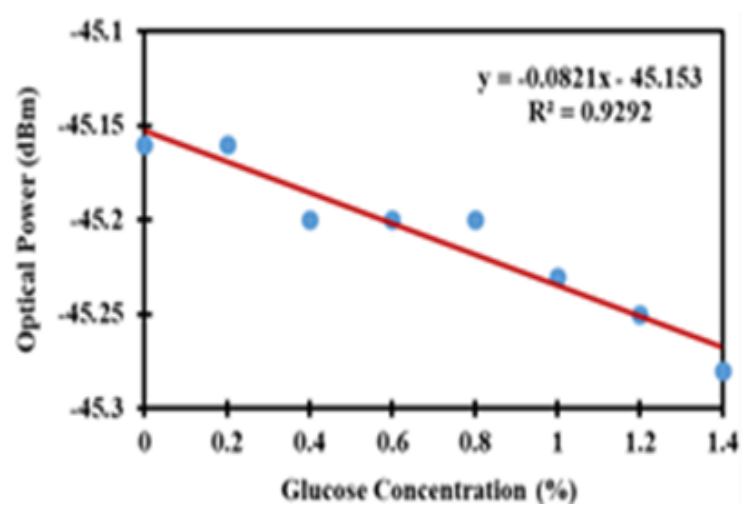

(b)

Figure 6. The sensing response; (a) sensing spectra and (b) analysis of reflected light intensity for the first cycle of the experiment at $1559 \mathrm{~nm}$ of wavelength for glucose solution range from $0 \%-0.14 \%$

Table 2. Optical fiber tip sensor performance at a medium range of glucose concentration

\begin{tabular}{cccc}
\hline No. of Cycles & 1 & 2 & 3 \\
\hline Sensor Sensitivity $(\mathrm{dBm} / \%)$ & 0.0821 & 0.1119 & 0.0911 \\
Regression Coefficient, R2 & 0.9292 & 0.9392 & 0.9552 \\
Average Sensitivity $(\mathrm{dBm} / \%)$ & & 0.0950 & \\
Average Linearity & & $94.21 \%$ & \\
\hline
\end{tabular}

\subsection{Sensing response for glucose concentration at low range of $0 \%$ to $0.14 \%$}

Figure 7 depicts the glucose sensing spectrum and the analysis of the reflected light with a glucose concentration ranging from $0 \%-0.14 \%$ for the first cycle of the experiment. As can be seen from the graph plotted in Figure 7(a), the sensing response from the proposed sensor does not distinguish the different 
glucose concentration. In the analysis of the optical output power, the regression line in Figure 7(b) only touches specific data points showing significant error terms. The linear regression model does not include all the possible variables.

The average sensor performance for the three cycles of the experiment is summarized in Table 3. The average linear regression for the repeated three cycles of the experiment is 0.0096 with linearity down to $9.6 \%$. This indicates that there is no significant changes in the output optical power and from the rough observation, the output optical power values are constant. The sensor sensitivity has a standard deviation of 0.089 , which strongly prove that the sensor sensitivity has variations and the results fluctuate for the three repeated experiments.

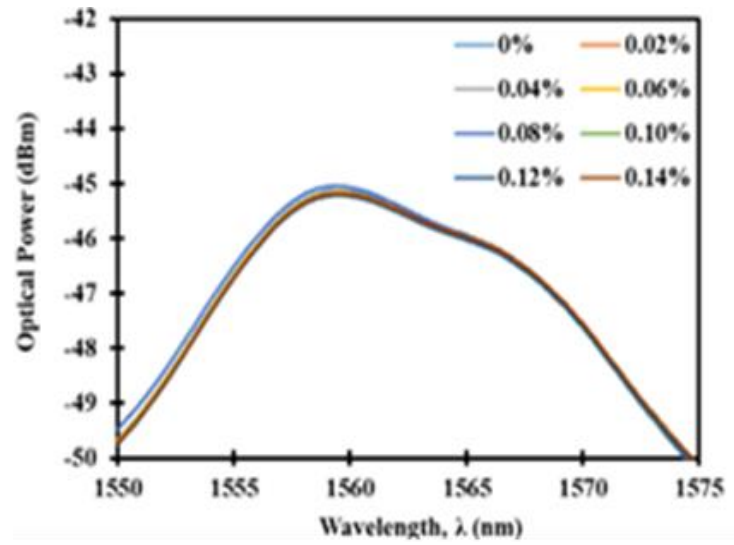

(a)

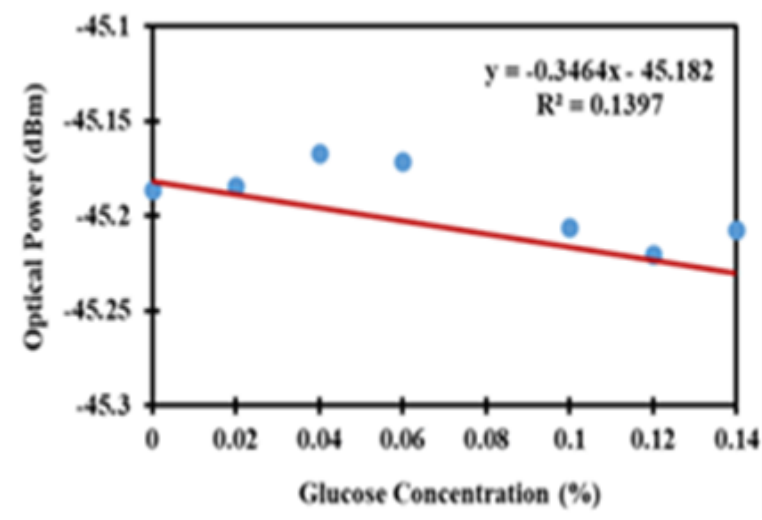

(b)

Figure 7. The sensing response; (a) sensing spectra and (b) analysis of reflected light intensity for the first cycle of the experiment at $1559 \mathrm{~nm}$ of wavelength for glucose solution range from $0 \%$ to $0.14 \%$

Table 3. Optical fiber tip sensor performance at the low range of glucose concentration

\begin{tabular}{cccc}
\hline No. of Cycles & 1 & 2 & 3 \\
\hline Sensor Sensitivity $(\mathrm{dBm} / \%)$ & 0.3464 & 0.2571 & 0.4357 \\
Regression Coefficient, R2 & 0.1397 & 0.0153 & 0.1330 \\
Average Sensitivity (dBm/\%) & & 0.3464 & \\
Average Linearity (\%) & & 9.6 & \\
Standard Deviation of the Sensitivity for Three Repeated Experiment, s & & 0.089 & \\
\hline
\end{tabular}

\subsection{Discussion}

To summarize the sensor performance, Table 4 tabulates all the results of the glucose sensing experiment. The sensor performance degrades as the glucose concentration changes from the higher to a lower range. Overall, the optical fiber tip sensor can be an ideal platform for glucose detection at a high and medium range of concentration. Generally, in both cases, the regression coefficient has a value of more than 0.90. The experimental data suit the fitted straight line for the concentration range of $0 \%-14 \%$ and $0 \%-$ $1.4 \%$ in three consecutive repeated experiment. This indicates a very strong correlation between the reflected optical power and glucose concentration at high and medium range. About $90 \%$ of the relationship is the result of the glucose concentrations which is the factor currently being considered in this activity, and promising good sensor linearity.

However, the linear relationship between the reflected optical power and glucose at the low range concentration shows a very weak correlation with no significant response. The insignificant changes may happen due to a very small changes in the concentration refractive index which cannot be detected by this sensor. Despite the fact that high sensitivity performance is obtained for the low range concentrations, this sensor has very low linearity and high standard deviations, proving that the sensor's severe problems were centered on the ability to differentiate between the smaller glucose concentration and the fact that it was incapable of measuring glucose at a lower concentration range. 
Table 4. Summary of the optical fiber tip sensor performance for glucose detection

\begin{tabular}{|c|c|c|c|}
\hline Glucose Range & $\begin{array}{l}\text { Average Sensor Sensitivity } \\
(\mathrm{dBm} / \%)\end{array}$ & Average Linearity (\%) & $\begin{array}{l}\text { Standard Deviation of the Sensitivity for } \\
\text { Three Repeated Experiment, s }\end{array}$ \\
\hline High Range (0\%-14\%) & 0.0979 & 99.27 & 0.000 \\
\hline Medium Range (0\%-1.4\%) & 0.0950 & 94.21 & 0.015 \\
\hline
\end{tabular}

\section{CONCLUSION}

This work has demonstrated the application of optical fiber tip sensor for glucose detection at a different range of concentration. The proposed sensor was tested with different glucose concentration ranges within a range; high $(0 \%-14 \%)$, medium $(0 \%-1.4 \%)$ and low $(0 \%-1.4 \%)$. The proposed sensor provides a strong sensitivity and linearity at high and medium concentrations with an average sensitivity of 0.0979 $\mathrm{dBm} / \%$ and $0.0950 \mathrm{dBm} / \%$, average linearity of $99.27 \%$ and $94.21 \%$, and standard deviations of 0.000 and 0.015 for the three repeated sensitivity results, respectively. However, the sensor has severe difficulties in detecting glucose at low concentration range; it fails to detect any significant changes during the measurement by providing a very high standard deviations for the three repeated sensitivity results as high as 0.089 and a very low average linearity as low as $9.6 \%$. Based on the findings obtained in this study, the optical fiber tip sensor has the potential to be utilized for glucose detection and suitable for high and medium concentration range measurement.

\section{ACKNOWLEDGEMENT S}

This work is financially supported by Universiti Teknologi Malaysia and Ministry of Higher Education (MoHE) under Research University Grant (RUG) vote Q.K130000.2543.19H84.

\section{REFERENCES}

[1] Malik, B.A. and M. Benaissa, "CHAPTER 5. Glucose Chemistry," in Dietary Sugars. The Royal Society of Chemistry. pp. 77-85, 2012.

[2] Dziedzic, S. and M. Kearsley, "Handbook of starch hydrolysis products and their derivatives," Springer Science \& Business Media, 2012

[3] Schlenker, E. and J.A. Gilbert, "Williams' Essentials of Nutrition and Diet Therapy-E-Book," Elsevier Health Sciences, 2014.

[4] Qadir, M.I. and H.N. Qureshi, "Is there any effect of blood glucose level on nail biting?," Journal of Medical Research and Health Sciences. vol. 2, no. 2, pp. 513-514, 2019.

[5] Thomas, S., et al., "Effectiveness of Structured Teaching Programme on Knowledge Regarding Hypoglycemia and its Management among Diabetic Patients," International Journal of Nursing Education, vol. 11, no. 1, pp. 140-143, 2019.

[6] Surgeons, A.A.o.O., "Advanced Emergency Care and Transportation of the Sick and Injured," Jones \& Bartlett Publishers, 2010.

[7] Wilson, A.M., et al., "HPLC Determination of Fructose, Glucose, and Sucrose in Potatoes," Journal of Food Science, vol. 46, no. 1, pp. 300-301, 1981.

[8] Parpinello, G.P. and A. Versari, "A simple high-performance liquid chromatography method for the analysis of glucose, glycerol, and methanol in a bioprocess," J Chromatogr Sci, vol. 38, no. 6, pp. 259-61, 2000.

[9] Zhang, H., et al., "Rapid determination of lactose, sucrose, glucose and fructose in foods by capillary zone electrophoresis with indirect ultraviolet detection," Chinese Journal of Chromatography, vol. 33, no. 8, pp. 816-821, 2015.

[10] Shen, Y.C., et al., "Determination of Glucose Concentration in Whole Blood using Fourier-Transform Infrared Spectroscopy," J Biol Phys, vol. 29, no. (2-3), pp. 129-33, 2003.

[11] Shakila, R.J., T. Vasundhara, K. Kumudavally, "A comparison of the TLC-densitometry and HPLC method for the determination of biogenic amines in fish and fishery products," Food Chemistry, vol. 75, no. 2, pp. 255-259, 2001.

[12] Adewusi, S.R. and J.H. Bradbury, "Carotenoids in cassava: Comparison of open-column and HPLC methods of analysis," Journal of the Science of Food and Agriculture, vol. 62, no. 4, pp. 375-383, 1993.

[13] Tan, R., et al., "Functionalized fiber end superstructure fiber bragg grating refractive index sensor for heavy metal ion detection," Sensors, vol. 18, no. 6, p. 1821, 2018.

[14] Yuan, J., et al., "A Fresnel reflection-based optical fiber sensor system for remote refractive index measurement using an OTDR," Photonic sensors, vol. 4, no. 1, pp. 48-52, 2014.

[15] Fujiwara, E., E. Ono, and C.K. Suzuki, "Application of an optical fiber sensor on the determination of sucrose and ethanol concentrations in process streams and effluents of sugarcane bioethanol industry," IEEE Sensors Journal, vol. 12, no. 9, pp. 2839-2843, 2012.

[16] Xu, W., X.G. Huang, and J.S. Pan, "Simple fiber-optic refractive index sensor based on fresnel reflection and optical switch,” IEEE Sensors Journal, vol. 13, no. 5, pp. 1571-1574, 2012. 
[17] Yoo, W.J., et al., "A fiber-optic sensor using an aqueous solution of sodium chloride to measure temperature and water level simultaneously," Sensors (Basel), vol. 14, no. 10, pp. 18823-36, 2014.

[18] Miya, T., et al., "Ultimate low-loss single-mode fibre at $1.55 \mu \mathrm{m}$," Electronics Letters, vol. 15, no. 4, pp. 106-108, 1979.

[19] Ohta, J., "Smart CMOS image sensors and applications," CRC press, 2007.

[20] Qiu, H., et al., "A novel graphene-based tapered optical fiber sensor for glucose detection," Applied Surface Science, vol. 329, pp. 390-395, 2015.

[21] Fu, M.-Y., et al., "Optical glucose sensor based on a fiber Bragg grating concatenated with a long period grating," in 2014 OptoElectronics and Communication Conference and Australian Conference on Optical Fibre Technology, IEEE, pp. 472-473, 2014.

[22] Kalantar-zadeh, K., "Sensors Characteristics, in Sensors: An Introductory Course," K. Kalantar-zadeh, Editor, Springer US: Boston, MA. pp. 11-28, 2013.

[23] Frankfort-Nachmias, C. and A. Leon-Guerrero, "Social Statistics for A Diverse Society. 4th ed," California: Pine Forge Press, 2006.

[24] Greener, I., "Designing social research: A guide for the bewildered," Sage Publications, 2011.

[25] Johnson, C.W., D.L. Timmons, and P.E. Hall, "Essential Laboratory Mathematics: Concepts and Applications for the Chemical and Clinical Laboratory Technician," 2nd ed. Canada: Delmar Learning, 2003.

[26] Yasin, M., et al., "Graphene coated silica microfiber for highly sensitive magnesium sensor," Sensors and Actuators A: Physical, vol. 273, pp. 67-71, 2018. 